\title{
Single item lot-sizing problem for a warm/cold process with immediate lost sales
}

\author{
Emre Berk ${ }^{*}$, Ayhan Özgür Toy ${ }^{1}$, Öncü Hazır ${ }^{1}$ \\ Faculty of Business Administration, Bilkent University, 06800 Ankara, Turkey
}

Received 31 January 2006; accepted 22 June 2006

Available online 21 November 2006

\begin{abstract}
We consider the dynamic lot-sizing problem with finite capacity and possible lost sales for a process that could be kept warm at a unit variable cost for the next period $t+1$ only if more than a threshold value $Q_{t}$ has been produced and would be cold, otherwise. Production with a cold process incurs a fixed positive setup cost, $K_{t}$ and setup time, $S_{t}$, which may be positive. Setup costs and times for a warm process are negligible. We develop a dynamic programming formulation of the problem, establish theoretical results on the structure of the optimal production plan in the presence of zero and positive setup times with Wagner-Whitin-type cost structures. We also show that the solution to the dynamic lot-sizing problem with lost sales are generated from the full commitment production series improved via lost sales decisions in the presence of a warm/cold process.
\end{abstract}

(C) 2006 Elsevier B.V. All rights reserved.

Keywords: Dynamic lot sizing; Warm/cold process; Lost sales; Setup times; Setup costs; Scheduling

\section{Introduction}

In this paper, we consider the dynamic lot-sizing problem with finite capacity and lost sales for a process that can be kept warm onto the next period at some variable cost provided that the production in the current period exceeds some given threshold. The process industries such as glass, steel and ceramic are the best examples where the physical nature of the production processes dictates that the processes be literally kept warm in certain periods to avoid expensive shutdown/startups. A striking instance comes from the glass industry. In some periods, production of glass is continued to avoid the substantial shutdown/startup costs and the produced glass is deliberately broken on the production line and fed back into the furnace. Similar practices are employed in foundries; ceramic and brick ovens are also kept warm sometimes even though no further production is done in the current period to avoid costly cooling-and-reheating procedures. Furthermore, Robinson and Sahin (2001) cite specific examples in food and petrochemical industries where certain cleanup and inspection

\footnotetext{
${ }^{*}$ Corresponding author. Fax: +90 3122664958.

E-mail addresses: eberk@bilkent.edu.tr (E. Berk), aotoy@bilkent.edu.tr (A.Ö. Toy), oncuh@bilkent.edu.tr (Ö. Hazır).

${ }^{1}$ Fax: +90 3122664958.
} 
operations can be avoided in the next period if the quantity produced in the current period exceeds a certain threshold so that the current production continues onto the next period. This may be done through either overtime or undertime. The treatment of the overtime option is outside the scope of our analysis; however, deliberate undertime practices can be studied within our context of warm/cold processes. With undertime, processes can be kept warm in environments with variable production rates by reducing the "nominal" or "calibrated" rate within a prespecified range (e.g., Silver, 1990; Moon et al., 1991; Gallego, 1993). As an illustration, suppose that the process is capable of producing at most $R$ units per time period at a nominal production rate and that its production rate can be reduced so that, within the same time period, the process can produce $Q(<R)$ units at the slowest rate. Thus, it is possible to keep this process warm by having it operate at rates lower than nominal so long as the quantity to be produced is between $Q$ and $R$. Such variable production rates are quite common in both process and discrete item manufacturing industries - feeder mechanisms can be adjusted so as to set almost any pace to a line; some chemical operations such as electroplating and fermentation can be decelerated deliberately (within certain bounds), and, manual operations can be slowed down by inserting idle times between units. Depending on the nature of the operations involved, the reduction in production rate can be obtained at either zero or positive additional cost. This additional variable cost is then the variable cost of keeping the process warm onto the next period. Aside from merely economic calculations, non-economic considerations such as safety of mounted tools and fixtures left idle on the machinery, impact on worker morale of engaging them in non-productive activities for a longer duration, impact of learning/forgetting phenomena on subsequent runs, etc. may also result in a managerial decision on a warm process threshold. The dynamic lot-sizing problem in the presence of production quantity-dependent warm/cold processes is a rather common problem; however, it has only recently been studied in Toy and Berk (2006). In their work, no shortages are allowed. In this work, we allow for possible lost sales. Before discussing the particulars of our work, we briefly review related works in the vast dynamic lot-sizing literature below.

The first formulation of the dynamic lot-sizing problem is independently by Wagner and Whitin (1958) and Manne (1958). The so-called Wagner-Whitin-Manne problem assumes a single item, uncapacitated production, no shortages and zero setup times. Wagner and Whitin (1958) provided a dynamic programming solution algorithm and structural results on the optimal solution of the classical problem which enable the construction of a forward solution algorithm. Recently, Aksen et al. (2003) extended the results of Wagner and Whitin to the case of immediate lost sales and showed that a forward polynomial algorithm is possible in this case, as well. When production capacity in a period is limited, the problem becomes the so-called capacitated lot-sizing problem (CLSP). The single-item CLSP has been shown to be NP-hard by Florian and Klein (1971). Certain special cases of the CLSP of horizon length $T$ have been shown to be solvable in polynomial time (Florian and Klein, 1971; Van Hoesel and Wagelmans, 1996; Bitran and Yanasse, 1982; Chung and Lin, 1988; Sandbothe and Thompson, 1990; Liu et al., 2004). For the CLSP for multi-item with positive setup times, we refer to a recent work, Gupta and Magnusson (2005). For a comprehensive review of the existing literature on the extension of the classical problem, we refer the reader to Wolsey (1995), Karimi et al. (2003) and Brahimi et al. (2006). Reformulations and algorithms for capacitated and uncapacitated lot-sizing problems are provided in Aggarwal and Park (1993) and Pochet and Wolsey (1995).

All of these works are different from the setting considered herein in that the production process is always cold, that is, it needs to be readied at positive cost for production in a particular period. The works that are close to our warm process setting are those in which it is possible to reserve a certain period for future production. This setting also occurs as a subproblem of the multi-item capacitated lot-sizing problem with the Lagrangian multipliers as the reservation costs for each of the periods and has been studied by Karmarkar et al. (1987), Eppen and Martin (1987), Hindi (1995a,b), and Agra and Constantino (1999). Although the models on lot sizing with reservation options employ the notion of a warm process, they do not consider a lower bound on the quantity produced for keeping the process warm onto the next period. We should also mention the related vast literature on the multi-item capacitated lot-sizing problems with sequence-dependent setups which consider warm processes but assumes only warm process thresholds of zero production. (See Allahverdi et al., 1999, this issue, for an extensive review on this subject.) Despite similarities, the above cited works are not readily applicable to our setting since we consider an explicit positive warm process threshold. Another body of work that uses the notion of warm processes is the discrete lot-sizing and scheduling problem (DLSP) literature (e.g. Fleischmann, 1990; Bruggemann and Jahnke, 2000; Loparic et al., 2003). This group of work differs from ours 
in the use of small bucket approach (i.e. $R=1$ in every period) and, more importantly, in that the process can be kept warm only if there has been capacitated production in the current period (i.e. $Q=R$ in every period). Thus, the results in the DLSP literature are not readily applicable to our general setting, as well. We should also mention the studies on lot sizing with undertime options. Moon et al. (1991), Silver (1990) and Gallego (1993) have examined the impact of using undertime via reduced production rates within the framework of multiple item lot scheduling problem with common cycles. In a recent work, Eiamkanchanalai and Banerjee (1999) also allows for bidirectional changes in the production rate. In all of these studies, continuous review and deterministic demand have been considered. As such, they do not reduce to our setting, either.

The problem studied in this paper builds on two recent works: Toy and Berk (2006) and Aksen et al. (2003). In the first work, the lot-sizing problem for a warm/cold process has been introduced for the first time and modeled as a dynamic programming problem. Specifically, they consider the dynamic lot-sizing problem with finite capacity where the process may be kept warm onto the next period at unit variable cost if production in the current period exceeds a threshold value; otherwise, the process is considered cold. Production in each period incurs variable production costs along with fixed setup costs. If the process is warm, a lower but positive setup cost is incurred. Both cold and warm setups are assumed to take no time. All demand must be satisfied in their periods. For this full commitment setting, the structural properties of the optimal policy have been identified and, based on them, it has been established that polynomial and linear time solution algorithms exist provided the Wagner-Whitin cost structure is maintained. In this study we consider a similar warm/cold process setting with zero costs and times for warm setups and provide a number of extensions of their work. First, we extend their formulation and results to the case of lost sales when there is no setup time. This model can also be viewed as an extension of Aksen et al. (2003) to a warm/cold process setting. Specifically, we show that forward solution algorithm also exists for our setting, as well. Second, in the presence of positive cold setup times, we obtain structural results regarding the optimal production plans and establish the conditions under which polynomial forward algorithms exist for the full commitment problem with a warm/cold process. Finally, based on the full commitment results, we establish the structural results and conditions for the lost sales problem. The rest of the paper is organized as follows: In Section 2, we present the basic assumptions of our model and formulate the optimization problem. In Section 3, we provide theoretical results on the structure of the optimal solution separately for the cases of zero and positive setup times. Finally, in Section 4, we provide some further numerical examples to highlight some key theoretical results.

\section{Model: Assumptions and formulation}

We have a single item. The length of the problem horizon, $N$ is finite and known. There are $£_{t}$ classes of demand in each period $t$. Different demand classes may occur in two ways: There may be different markets in which different prices may be charged for the item; or, the item may be a component that is used internally for different products. The amount of demand of class $\ell$ in period $t$ is denoted by $D_{t}^{\ell}(t=$ $1,2, \ldots, N ; \ell=1,2, \ldots, £_{t}$ ). (In the sequel $D_{t}^{\ell}$ will be used interchangeably to denote the set and cardinality of demand class, as it will be clear from the context.) All demands are non-negative and known, but may be different over the planning horizon. The amount of production in period $t$ is denoted by $x_{t}$. For every item produced in period $t$, a unit production $\operatorname{cost} c_{t}$ is incurred. The inventory on hand at the end of period $t$ is denoted by $y_{t}$; an inventory holding $\operatorname{cost} h_{t}$ is incurred for every unit of ending inventory in the period. Without loss of generality (w.l.o.g.), we assume that the initial inventory level is zero. Each demand of class $\ell$ that is satisfied brings in a revenue of $p_{t}^{\ell}$. We make no assumption regarding the structure of revenues other than that the gross marginal profit $\left(p_{t}^{\ell}-c_{t}\right)$ is non-negative for all $\ell$ and $t$. The decision maker has the option of not satisfying a particular demand, in which case it is lost. With each unit of lost sales, only the revenue is foregone and no additional cost is incurred; furthermore, future demands are not affected by the lost sales decision in any period. The time during which the process can be used in a period is bounded by non-negative with a maximum capacity, $R_{t}$. We consider warm and cold production processes defined as follows: the production process may be kept warm onto the beginning of period $t$ if the total process time in period $t-1$ is at least $Q_{t-1}$; otherwise, the process cannot be kept warm and is cold. We use the term process time to denote the total of production time and setup times, if applicable. W.l.o.g., the nominal unit production time is one time unit for any period $t$ such that the production quantity $x_{t}$ can also be used interchangeably as the production time in 
period $t$ if the nominal production rate is used. A cold process requires a cold setup with an incurred cost $K_{t}$, if production is to be done in period $t$. A cold setup also takes up setup time $S_{t}$, which may be zero or positive. A warm process requires no setup; that is, warm setup times and costs are zero. For example, in glass manufacturing, keeping the furnaces warm essentially ensures that production in the next period starts with no setup. Other practical applications include a production line whose physical layout or a machine whose calibration is maintained for the next period at no or almost no additional fixed cost. To keep the process warm onto period $t, \omega_{t-1}$ is charged for every unit of unused capacity in period $t-1$. That is, the warming cost incurred in period $t-1$ would be $\omega_{t-1}\left(R_{t-1}-x_{t-1}-S_{t-1}\right)$ monetary units if a cold setup had been done in that period, and $\omega_{t-1}\left(R_{t-1}-x_{t-1}\right)$ monetary units if a warm setup had been done in that period. Note that even if the process time in period $t-1$ is at least $Q_{t-1}$, it may not be optimal to keep the process warm onto the next period if during the next period there would not be any production; in such instances, there will be no warming cost incurred. Observe that the existence of a warm threshold implicitly suggests that $K_{t}>\omega_{t-1}\left(R_{t-1}-Q_{t-1}\right) \forall t$.

The objective is to find a production plan $(X, A, \Gamma)$ that consists of the production schedule $x_{t} \geqslant 0$ (timing and amount of production) and the amount of lost sales $a_{t}^{\ell}$ of class $\ell$ and $\gamma_{t}$ (a binary variable indicating a cold setup decision in period $t)(t=1,2, \ldots, N)$ such that total profit over the horizon is maximized. (In the sequel we shall use the shorthand $\mathbf{a}_{t}$ and $\mathbf{D}_{t}$ to denote respectively the arrays of lost sales and demands for all demand classes for period $t$. Furthermore, for brevity, we shall suppress $\Gamma$ unless needed explicitly and use $(X, A)$ to denote a production plan in the sequel.) Let $y_{t-1}$ be the starting inventory, $z_{t}$ be a binary variable indicating the cold status of the process, and $\delta_{t}$ be a binary variable indicating positive production for period $t$. Then, the total profit for the entire horizon can be written as

$$
\Pi=\sum_{t=1}^{N} \sum_{\ell=1}^{\mathfrak{f}_{t}} p_{t}^{\ell} D_{t}^{\ell}-\sum_{t=1}^{N} \sum_{\ell=1}^{\mathfrak{£}_{t}}\left(K_{t} \gamma_{t}+\left[\omega_{t-1}\left[R_{t-1}-x_{t-1}-\gamma_{t-1} S_{t-1}\right]^{+} \cdot\left(1-\gamma_{t}\right)\right]+c_{t} x_{t}+h_{t} y_{t}+p_{t}^{\ell} a_{t}^{\ell}\right) .
$$

Hence, the problem of maximizing the total profit $\Pi$ is equivalent to problem $(P)$ of minimizing the total cost minus the total revenue that would have been received if all demands were satisfied, $\sum_{t=1}^{N} \sum_{\ell=1}^{\mathfrak{\ell}_{t}} p_{t}^{\ell} D_{t}^{\ell}$. In the sequel, we work with the equivalent problem $P$, and, for brevity, we shall drop the constant summation term. We develop a dynamic programming formulation of the problem $(P)$. Let $f_{t}^{N}\left(x_{t-1}, y_{t-1}, \gamma_{t-1}\right)$ denote the minimum total cost under an optimal production schedule for periods $t$ through $N$. Then,

$$
\begin{aligned}
f_{t}^{N}\left(x_{t-1}, y_{t-1}, \gamma_{t-1}\right)= & \min _{\substack{x_{t}, \mathbf{a}_{t}, \gamma_{t} \\
0 \leqslant x_{t}+\gamma_{t} S_{t} \leqslant R_{t}}}\left[K_{t} \gamma_{t}+\left[\omega_{t-1}\left[R_{t-1}-x_{t-1}-\gamma_{t-1} S_{t-1}\right]^{+} \cdot\left(1-\gamma_{t}\right)\right]+c_{t} x_{t}+h_{t} y_{t}\right. \\
& x_{t}+y_{t-1}+\sum_{\ell=1}^{\mathfrak{t}_{t}} a_{t}^{\ell} \geqslant \sum_{\ell=1}^{t_{t}} D_{t}^{\ell} \\
+ & \left.\sum_{\ell=1}^{\mathfrak{t}_{t}} p_{t}^{\ell} a_{t}^{\ell}+f_{t+1}^{N}\left(x_{t}, y_{t}, \gamma_{t}\right)\right],
\end{aligned}
$$

where

$$
\begin{aligned}
& y_{t}=y_{t-1}+x_{t}+\sum_{\ell=1}^{\mathfrak{£}_{t}} a_{t}^{\ell}-\sum_{\ell=1}^{\mathfrak{f}_{t}} D_{t}^{\ell} \quad \text { for } t=1,2, \ldots, N \\
& \gamma_{t}=\left\{\begin{array}{ll}
0 & \text { otherwise } \\
1 & \text { if } x_{t}>0 \text { and } x_{t-1}<Q_{t-1}-\gamma_{t-1} S_{t-1}
\end{array} \text { for } t=1,2, \ldots, N\right.
\end{aligned}
$$

with the boundary condition in period $N$ :

$$
\begin{aligned}
& f_{N}^{N}\left(x_{N-1}, y_{N-1}, \gamma_{N-1}\right)=\min _{\substack{x_{N}, \mathbf{a}_{N}, \gamma_{N} \\
0 \leqslant x_{N}+\gamma_{N} S_{N} \leqslant R_{N}}}\left[\mathfrak{E}_{N} \gamma_{N}+\gamma_{N}+\omega_{N-1}\left[R_{N-1}-x_{N-1}-\gamma_{N-1} S_{N-1}\right]^{+} \cdot\left(1-\gamma_{N}\right)\right] \\
& \left.+c_{N} x_{N}+h_{N} y_{N}+\sum_{\ell=1}^{\mathfrak{E}_{N}} p_{N}^{\ell} a_{N}^{\ell}\right] \text {. }
\end{aligned}
$$


The optimal solution is found using the above recurrence and $f_{1}^{N}(0,0,0)$ denotes the minimum cost for periods 1 through $N$ where we arbitrarily set $x_{0}=0, \gamma_{0}=0$. W.1.o.g., we assume throughout that $y_{0}=y_{N}=0$ and, for convenience, $R_{0}=S_{0}=\omega_{0}=0$ and that all demands and capacities are integer valued. The above DP formulation makes no assumptions about the cost parameters, demand pattern and production capacities. It is solvable in pseudo-polynomial time with $\mathrm{O}\left(2 N^{2} R \bar{d}\right)$ where $\bar{d}$ denotes the average demand per period and $R=\max \left\{R_{t}\right\}$. It is desirable to obtain certain additional conditions to reduce the solution space and computational effort. We next examine some of the structural properties of the optimal solution to this end.

\section{Structural results}

In this section, we present structural results on the optimal production plan for the lot-sizing problem with a warm/cold process. In particular, we establish the conditions under which production is to be done and the amount of production in a period. Furthermore, we show that certain production plans enable one to solve the original problem by considering independently solvable subproblems.

We first establish the equivalence property between a problem $P$ in which there are periods with demands exceeding the production capacities in those periods and another modified problem $P^{\prime}$ in which no demand is greater than the capacity.

Proposition 1 (Problem equivalence). If problem $P$ is feasible, it can be re-written as an equivalent problem where in each period the demand is not greater than the capacity.

Proof is in Appendix. Hence, without loss of generality, we assume in the sequel that $\sum_{\ell=1}^{\mathfrak{f}_{t}} D_{t}^{\ell} \leqslant R_{t}$ for all $t$. In the sequel we make a number of additional assumptions. We assume that physical capacities are nondecreasing, i.e. $R_{t-1} \leqslant R_{t}$ for all $t$. Additionally, we assume that all cold setup costs are non-negative, with $K_{t} \geqslant K_{t+1}$ for all $t$. Finally, regarding costs, we assume that (i) $c_{t}+h_{t}-\omega_{t}>c_{t+1}$. Consequently, (ii) $c_{t}+h_{t}>c_{t+1}$ and (iii) $c_{t}+h_{t}-\omega_{t}>c_{t+1}-\omega_{t+1}$. These conditions are similar to those in Toy and Berk (2006) and ensure that Wagner-Whitin-type costs are incurred for productions not exceeding the warm thresholds in both of the consecutive periods, for productions exceeding the threshold in one but not in the other, and for productions exceeding in both of the consecutive periods, respectively. As will be shown in the sequel, this cost structure prohibits any speculative production behavior, that is, ensures producing for a particular period's demands in a period as close to it as possible. (Later, we also consider a special case when $c_{t}+h_{t}-\omega_{t}<c_{t+1}$.)

We analyze the problem separately for the cases of zero and positive setup times. But we introduce a common notation. Let $(\mathbf{X}, \mathbf{A})=\left\{\left\{x_{1}, \ldots, x_{N}\right\},\left\{\mathbf{a}_{1}, \ldots, \mathbf{a}_{N}\right\}\right\}$ denote a feasible production plan constructed over periods 1 through $N$.

Let $\Psi_{u v \mid I_{u-1}, I_{v-1}}$ represent a subset of $X$ between two consecutive cold setups in periods $u$ and $v$ with given starting and ending inventories such that $y_{u-1}=I_{u-1}$ and $y_{v-1}=I_{v-1}$. We have $\Psi_{u v \mid I_{u-1}, I_{v-1}}=$ $\left\{x_{i} \mid x_{i}>0, i=u, \ldots, m ; x_{i}=0\right.$ for $i=m+1, \ldots, v-1 ; \mathbf{a}_{i} \geqslant \mathbf{0} ; z_{u}=1=z_{v} ; z_{i}=0$ for $u+1 \leqslant i \leqslant m ; z_{m+1} \geqslant 0$ and $z_{i}=1$ for $\left.m+2 \leqslant i \leqslant v-1\right\}$ where $m$ denotes the latest period in which production is done between $u$ and $v-1$ for $0<u \leqslant m<v \leqslant N+1$. We will call $\Psi_{u v \mid I_{u-1}, I_{v-1}}$ a production series, and a period $t$ for $u+1 \leqslant t \leqslant m-1$ will be called an intermediate production period. Note that, a production series may begin and end with positive inventory, i.e. $I_{u-1} \geqslant 0, I_{v-1} \geqslant 0$.

\subsection{Zero setup times}

In this subsection, we restrict our analysis to the case where $S_{t}=0 \forall t$. We proceed in two steps. We first consider the problem where no lost sales are allowed and discuss certain properties of it. Then, we introduce the (possible) lost sales decisions and establish structural results for $P$. These structural properties can be used to modify the problem $P$, given in (2)-(5) to reduce the search space and effort, and also to develop a forward solution algorithm.

We define a full commitment problem to be problem $P$ under the additional constraint that no lost sales are allowed i.e., $\mathbf{a}_{t} \equiv \mathbf{0} \forall t$ and denote it with $\widetilde{P}$. Similarly, a production series $\Psi_{u v \mid I_{u-1}, I_{v-1}}$ will be called a full commitment series if we set a priori $\mathbf{a}_{i} \equiv \mathbf{0}$ for $u \leqslant i \leqslant v-1$. Toy and Berk (2006) analyze a full 
commitment problem where there may be positive fixed warm setup costs. As such, the full commitment problem $\widetilde{P}$ herein which has zero warm setup costs is a special case contained in the afore mentioned paper. Hence, we adopt their definitions and invoke some of their results.

Lemma 1. An optimal production plan for problem $\widetilde{P}$ has the property $\gamma_{t}=\delta_{t} \cdot z_{t}$ and $\gamma_{t} \cdot y_{t-1}=0$ for $t=1,2, \ldots, N$ where $\gamma_{t}$, and $y_{t-1}$ are as given in (3) and (4), $\delta_{t}=1$ if $x_{t}>0$ and 0 otherwise, and $z_{t}=1$ if $x_{t-1}+\gamma_{t-1} S_{t-1}<Q_{t-1}$ and 0 otherwise.

Lemma follows from Theorem 4(ii) in Toy and Berk (2006). The first property implies that there is no cold setup in a period in which either there is no production or the process is warm. The second property implies that there is no cold setup in a period in which the beginning inventory is positive. Hence, the starting and ending inventories for any production series in a full commitment problem reduce to zero. Therefore, in the analysis below, we consider only series $\Psi_{u v \mid 0,0}$, which we will refer to in shorthand as $\Psi_{u v}$.

In addition to the physical capacity in period $t$, we define an economic bound, $E_{t}$, on the production quantity in the presence of warm/cold processes. $E_{t}$ is such a quantity that producing more than this quantity in period $t$ for a future period is more costly than producing the excess quantity in period $t+1$ while satisfying the net demand for period $t$ and keeping the process warm for period $t+1$. Thus,

Lemma 2 (Lemma 1(i) in Toy and Berk, 2006). In an optimal production plan for problem $\widetilde{P}, x_{t} \leqslant E_{t}$ where

$$
E_{t}=\frac{\max \left(Q_{t},\left[\sum_{\ell=1}^{\mathfrak{f}_{t}} D_{t}^{\ell}-y_{t-1}\right]^{+}\right) \cdot\left(c_{t}+h_{t}-c_{t+1}-\omega_{t}\right)+R_{t} \omega_{t}}{c_{t}+h_{t}-c_{t+1}} \quad \forall t .
$$

Next, we provide the structure of a production series in an optimal production plan for the full commitment problem.

Theorem 1 (Optimal production quantity theorem; Theorem 4(i) in Toy and Berk, 2006). In an optimal solution to problem $\widetilde{P}$, each production series $\Psi_{\text {uv }}$ is of the form:

(a) $x_{t}=\max \left(Q_{t},\left[\sum_{\ell=1}^{\mathfrak{f}_{t}} D_{t}^{\ell}-y_{t-1}\right]^{+}\right)$, for $u \leqslant t \leqslant m-1$ and $m-u>0$,
(b) $x_{m}=\left[\sum_{i=m}^{v-1} \sum_{\ell=1}^{\mathfrak{£} i} D_{i}^{\ell}-y_{m-1}\right]^{+}<E_{m}$ and $m-u \geqslant 0$.

It is important to note that there are some fundamental differences between the (feasibility ensured equivalent) CLSP (under Proposition 1 in Bitran and Yanasse, 1982) and $\widetilde{P}$ with zero setup times. In CLSP for a single item in the literature, there is only one setup type - cold setup, whereas in $\widetilde{P}$ considered in our work there is also a warm setup possibility. With a slight abuse of notation, we can say that, in $\widetilde{P}, Q_{t} \leqslant R_{t}$ but, in CLSP, $Q_{t}$ is strictly greater than $R_{t}$, which prohibits any warm setup possibility. Also, it may be optimal to produce even in a period of zero demand in our setting unlike the CLSP (see Corollary 2.1 in Bitran and Yanasse, 1982). Furthermore, in the classical uncapacitated problem setting, the positive production quantity in any period is exactly equal to a sum of demands for a finite number of periods into the future. In our setting, this property holds not for individual production quantities but for the quantity produced in the entire production series. Finally, in CLSP, the optimal production plan is composed of subplans in which the production quantities in any period are either zero or at capacity, except for at most one period in which it is less than capacity. In our setting, this property no longer holds.

So far, we have only considered the properties of problem $\widetilde{P}$. Building on these, we next consider the introduction of lost sales for zero setup times. In the following, we use $\widehat{\Psi}_{u v}$ to denote a full commitment series $\Psi_{u v}$ after its modification with possible lost sales decisions for the periods between $u$ and $v-1$; we call $\widehat{\Psi}_{u v}$ a lostsales-improved series. Note that a lost-sales-improved series may be identical to a full commitment series if it is optimal not to lose any sales. Also note that a lost-sales-improvement can be such that all demands in periods $u$ through $v-1$ are lost; that is $x_{i}=0$ and $a_{i}^{\ell}=D_{i}^{\ell}$ for $u \leqslant i \leqslant v-1$. We call such a series a null series. For brevity, we shall abuse the introduced series notation slightly and continue to use period $u$ as the beginning 
period of the series (and also the ending period of the previous series) even though there is no longer any production (and, hence a cold setup) in that period to remind us that $\widehat{\Psi}_{u v}$ has been obtained by considering a full commitment series between periods $u$ and $v$. This slight abuse of notation allows us to construct the optimal plan of problem $P$ by first considering full commitment series and, then, improving them through lost sales and, finally, by patching up lost-sales-improved series. As we show below, a lost-sales-improved series is obtained by applying the improvement rules iteratively. In each iteration, one demand class per period is considered. Specifically, we envision the following procedure. Start with series $\Psi_{u v}$. Identify the demand class $\ell$ and period $j$ that improves the costs if lost; construct the lost-sales-improved series $\widehat{\Psi}_{u v}$. Clearly, $\widehat{\Psi}_{u v}$ has the identical production schedule as another full commitment series $\Psi_{u v}^{\prime}$ for the demand pattern $\mathbf{D}^{\prime}$ such that $D_{j}^{\ell \prime}=D_{j}^{\ell}-a_{j}^{\ell}$ with the rest of demands being as before. Now, apply the same rules on $\Psi_{u v}^{\prime}$ and obtain $\widehat{\Psi}_{u v}^{\prime}$; and repeat until there is no improvement over the segment consisting periods $u$ through $v$. Now, we proceed to the properties of problem $P$.

The first result we present below states that in $P$, demands in period $t$ can be lost if and only if there is no production in this period.

Theorem 2 (Lost sales theorem). In an optimal production plan for problem $P, x_{t} \cdot a_{t}^{\ell}=0$ for $\ell=1,2, \ldots, £_{t}$ $\forall t$.

The proof is by contradiction (Berk et al., 2006). The above result reduces to Lemma 1 in Aksen et al. (2003) for a single demand class and infinite capacity in each period. From Theorems 1 and 2, we have

Corollary 1. In a lost-sales-improved production series $\widehat{\Psi}_{u v}, \mathbf{a}_{j}=\mathbf{0}$ if $x_{u}>0$ for $u \leqslant j \leqslant m$. Conversely, a may be positive only for $m<j<v, \exists \ell$.

Remark. It follows from the above corollary that $\widehat{\Psi}_{u v}$ can be obtained from a full commitment production series. To achieve this, one needs only to consider lost sales decisions for demands in periods where there is no production done (i.e., $m+1$ through $v-1$ ). That is, a full commitment production series $\Psi_{u v}$, in which the latest production period is $m$, may be modified by the introduction of lost sales only by decreasing the overall quantity produced starting from period $m$ backwards to yield a lost-sales-improved production series $\widehat{\Psi}_{u v}$. The construction of $\widehat{\Psi}_{u v}$ is as follows. In a least cost full commitment series $\Psi_{u v}$ for a given demand pattern $\mathbf{D}_{t}$ for $u \leqslant t \leqslant v-1$, the ending inventory in period $m(u \leqslant m<v)$ is given by $y_{m}=\sum_{i=m+1}^{v-1} \sum_{\ell=1}^{f i} D_{i}^{\ell}$. Now, suppose that the demand pattern is modified as $\mathbf{D}_{t}^{\prime}=\mathbf{D}_{t}-\mathbf{a}_{t} \forall t$ such that $0<\sum_{i=m+1}^{v-1} \sum_{\ell=1}^{\mathfrak{f} i} a_{i}^{\ell}$ and $\mathbf{a}_{t}=\mathbf{0}$ for $u \leqslant t \leqslant m$. Let $\Psi_{u v}^{\prime}$ denote the new least cost full commitment series for this modified demand pattern. If

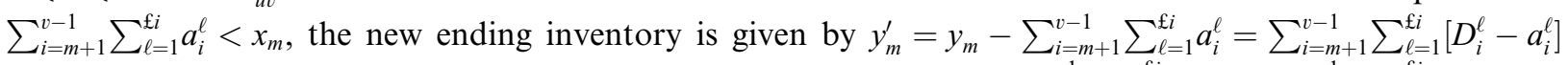

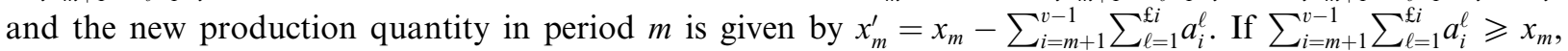
in the new series, the latest production is done in period $m^{\prime}(<m)$ and the ensuing production series structure is as given by Theorem 1 . This remark provides us with an improvement rule that we elaborate as we develop a forward solution algorithm below.

Theorem 3 (Lost-sale-improvement theorem).

(i) If $c_{m}+\sum_{i=m}^{j-1} h_{i}>p_{j}^{\ell}$, then a full commitment series $\Psi_{u v}$ can be improved by a lost sales decision $\hat{a}_{j}^{\ell}(>0)$ for some demand class $\ell$ in period $j(>m)$ where $0<\hat{a}_{j}^{\ell} \leqslant \min \left(D_{j}^{\ell}, x_{m}\right)$.

(ii) If $p_{j}^{\ell} \geqslant c_{m}+\sum_{i=m}^{j-1} h_{i}$, then a full commitment series $\Psi_{u v}$ cannot be improved by a lost sales decision $\hat{a}_{j}^{\ell}(>0)$ for some demand class $\ell$ in period $j(>m)$ where $0<\hat{a}_{j}^{\ell} \leqslant \min \left(D_{j}^{\ell}, x_{m}-\left[E_{m-1}-x_{m-1}\right]\right)$.

(iii) Let $\bar{j}(\ell)$ denote the earliest period in which some of demand class $\ell$ of period $j(>m)$ is produced; $\bar{j}(\ell)=\max \left\{t: \sum_{i=t}^{m} x_{i} \geqslant D_{j}^{\ell}\right\}$. If $c_{\bar{j}(\ell)}+\sum_{i=\bar{j}(\ell)}^{j-1} h_{i}<p_{j}^{\ell}$, then a full commitment series $\Psi_{u v}$ cannot be improved by a lost sales decision $\hat{a}_{j}^{\ell}(>0)$.

(iv) Any full commitment series $\Psi_{u v}$ can be improved by a null series if foregoing the cold setup and losing all demands yield a lower cost.

Theorem 4 (Lose all or nothing theorem). In an optimal production plan for problem $P, a_{t}^{\ell} \cdot\left[D_{t}^{\ell}-a_{t}^{\ell}\right]=0$ for $\ell=1,2, \ldots, £_{t} \forall t$. 
The proofs for the above theorems are by contradiction (Berk et al., 2006). With the above results, we have shown the properties of the optimal production and lost sales decisions for periods within a segment of the problem consisting of periods $u$ through $v-1(1 \leqslant u \leqslant v-1 \leqslant N)$ where a cold setup is done in period $u$ to produce (all or some of) the demands for periods for $u$ through $v-1$ in a continuous production stream. Since these properties hold for any segment $[u, v)$, the optimal solution for problem $P$ must consist of a subset of all such series constructed. Therefore, we conclude that the optimal solution for problem $P$ can be found by considering only the series the construction of which obey the properties developed above, which we state formally:

Lemma 3. The optimal solution for $P$ where $S_{t}=0 \forall t$ consists only of series that are the lost-sales-improvements of the series for $\widetilde{P}$ with $S_{t}=0 \forall t$.

We provide an example to illustrate the properties of the optimal solution.

Example. Consider a problem horizon of five periods with the given demands $\{42,20,40,40,65\}$. For all $t$, $S_{t}=0, R_{t}=100, Q_{t}=70, K_{t}=110, h_{t}=1, \omega_{t}=0.85, c_{t}=0$, and $p_{t}=2$. We use the notation $\mathbf{X}_{N}$ and $\mathbf{A}_{N}$ to denote the sets of production schedules and lost sales decisions considered for a problem of $N$ periods with $X_{N}^{*}$ denoting the optimal production plan for it. We first illustrate below the forward solution methodology for the full commitment problem, $\widetilde{P}$. (Note that we have $\mathbf{A}=\mathbf{0}$ throughout.)

$$
\begin{aligned}
& N=1 ; \quad \Psi_{1,1}=\{42\} ; \mathbf{X}_{1}=\left\{\Psi_{1,1}\right\} ; X_{1}^{*}=\Psi_{1,1} ; f_{1}^{1}=110, \\
& N=2 ; \quad \Psi_{1,2}=\{62,0\}, \Psi_{2,2}=\{20\} ; \mathbf{X}_{2}=\left\{\Psi_{1,2},\left(X_{1}^{*} \cup \Psi_{2,2}\right)\right\} ; X_{2}^{*}=\Psi_{1,2} ; f_{1}^{2}=130, \\
& N=3 ; \quad \Psi_{1,3}=\{70,32,0\}, \Psi_{2,3}=\{60,0\}, \Psi_{3,3}=\{40\} ; \\
& \mathbf{X}_{3}=\left\{\Psi_{1,3},\left(X_{1}^{*} \cup \Psi_{2,3}\right),\left(X_{2}^{*} \cup \Psi_{3,3}\right)\right\} ; X_{3}^{*}=\Psi_{1,3} ; f_{1}^{3}=203.5, \\
& N=4 ; \quad \Psi_{1,4}=\{70,72,0,0\}, \Psi_{2,4}=\{70,30,0\}, \Psi_{3,4}=\{80,0\}, \Psi_{4,4}=\{40\} ; \\
& \mathbf{X}_{4}=\left\{\Psi_{1,4},\left(X_{1}^{*} \cup \Psi_{2,4}\right),\left(X_{2}^{*} \cup \Psi_{3,4}\right),\left(X_{3}^{*} \cup \Psi_{4,4}\right)\right\} ; X_{4}^{*}=\left(X_{2}^{*} \cup \Psi_{3,4}\right)=\{62,0,80,0\} ; f_{1}^{4}=280, \\
& N=5 ; \quad \Psi_{1,5}=\{70,70,67,0,0\}, \Psi_{2,5}=\{70,95,0,0\}, \Psi_{3,5}=\{70,75,0\}, \Psi_{4,5}=\{70,35\}, \Psi_{5,5}=\{65\} ; \\
& \mathbf{X}_{5}=\left\{\Psi_{1,5},\left(X_{1}^{*} \cup \Psi_{2,5}\right),\left(X_{2}^{*} \cup \Psi_{3,5}\right),\left(X_{3}^{*} \cup \Psi_{4,5}\right),\left(X_{4}^{*} \cup \Psi_{5,5}\right)\right\} ; X_{5}^{*}=\left(X_{2}^{*} \cup \Psi_{3,5}\right)=\{62,0,70,75,0\} ; f_{1}^{5}=360.5 .
\end{aligned}
$$

Next, we illustrate the forward solution methodology for problem $P$. In its solution we shall use the full commitment series developed above and improve them through lost sales decisions if possible. In cases of indifference, we use the lost-sales-improved series. We do not report $\mathbf{A}_{N}$ for brevity which can be obtained easily from $\mathbf{X}_{N}$

$$
\begin{aligned}
& N=1 ; \quad \Psi_{1,1}=\{42\} ; \widehat{\Psi}_{1,1}=\{0\} ; \mathbf{X}_{1}=\left\{\widehat{\Psi}_{1,1}\right\} ; X_{1}^{*}=\widehat{\Psi}_{1,1} ; f_{1}^{1}=84, \\
& N=2 ; \quad \Psi_{1,2}=\{62,0\}, \Psi_{2,2}=\{20\} ; \widehat{\Psi}_{1,2}=\{62,0\}, \widehat{\Psi}_{2,2}=\{0\} ; \mathbf{X}_{2}=\left\{\widehat{\Psi}_{1,2},\left(X_{1}^{*} \cup \widehat{\Psi}_{2,2}\right)\right\} ; \\
& X_{2}^{*}=\left(X_{1}^{*} \cup \widehat{\Psi}_{2,2}\right)=\{0,0\} ; f_{1}^{2}=124, \\
& N=3 ; \quad \Psi_{1,3}=\{70,32,0\}, \Psi_{2,3}=\{60,0\}, \Psi_{3,3}=\{40\} ; \widehat{\Psi}_{1,3}=\{70,32,0\}, \widehat{\Psi}_{2,3}=\{0,0\}, \widehat{\Psi}_{3,3}=\{0\} ; \\
& \mathbf{X}_{3}=\left\{\widehat{\Psi}_{1,3},\left(X_{1}^{*} \cup \widehat{\Psi}_{2,3}\right),\left(X_{2}^{*} \cup \widehat{\Psi}_{3,3}\right)\right\} ; X_{3}^{*}=\widehat{\Psi}_{1,3} ; f_{1}^{3}=203.5, \\
& N=4 ; \quad \Psi_{1,4}=\{70,72,0,0\}, \Psi_{2,4}=\{70,30,0\}, \Psi_{3,4}=\{80,0\}, \Psi_{4,4}=\{40\} ; \\
& \widehat{\Psi}_{1,4}=\{70,32,0,0\}, \widehat{\Psi}_{2,4}=\{0,0,0\}, \widehat{\Psi}_{3,4}=\{80,0\}, \widehat{\Psi}_{4,4}=\{0\} ; \\
& \mathbf{X}_{4}=\left\{\widehat{\Psi}_{1,4},\left(X_{1}^{*} \cup \widehat{\Psi}_{2,4}\right),\left(X_{2}^{*} \cup \widehat{\Psi}_{3,4}\right),\left(X_{3}^{*} \cup \widehat{\Psi}_{4,4}\right)\right\} ; X_{4}^{*}=\left(X_{2}^{*} \cup \widehat{\Psi}_{3,4}\right)=\{0,0,80,0\} ; f_{1}^{4}=274, \\
& N=5 ; \quad \Psi_{1,5}=\{70,70,67,0,0\}, \Psi_{2,5}=\{70,95,0,0\}, \Psi_{3,5}=\{70,75,0\}, \Psi_{4,5}=\{70,35\}, \Psi_{5,5}=\{65\} ; \\
& \widehat{\Psi}_{1,5}=\{0,0,0,0,0\}, \widehat{\Psi}_{2,5}=\{0,0,0,0\}, \widehat{\Psi}_{3,5}=\{70,75,0\}, \widehat{\Psi}_{4,5}=\{70,35\}, \widehat{\Psi}_{5,5}=\{65\} ; \\
& \mathbf{X}_{5}=\left\{\widehat{\Psi}_{1,5},\left(X_{1}^{*} \cup \widehat{\Psi}_{2,5}\right),\left(X_{2}^{*} \cup \widehat{\Psi}_{3,5}\right),\left(X_{3}^{*} \cup \widehat{\Psi}_{4,5}\right),\left(X_{4}^{*} \cup \widehat{\Psi}_{5,5}\right\} ; X_{5}^{*}=\left(X_{2}^{*} \cup \widehat{\Psi}_{3,5}\right)=\{0,0,70,75,0\} ; f_{1}^{5}=354.5 .\right.
\end{aligned}
$$

\subsection{Positive setup times}

In the previous subsection, we have only considered the case where $S_{t}=0$. Now, we relax this restriction so that $0<S_{t} \leqslant R_{t}$. In this case, the process time in period $t$ consists of the time spent for production in that period and the setup time if a cold setup is incurred in the period. Our goal remains to be identifying certain con- 
ditions that enable us to develop optimal production plans for problem $P$ through lost-sales-improvements of the series for problem $\widetilde{P}$ with $S_{t}>0 \forall t$. Without loss of generality, we assume for brevity that $\sum_{\ell=1}^{\mathfrak{f}_{1}} D_{t}^{\ell} \leqslant R_{t}-S_{t}$ for the first period $(t=1)$; otherwise, the demands would have to be lost and an equivalent solution can be obtained.

The introduction of positive cold setup times changes certain structural characteristics of the problem vis $a$ vis zero setup times case. The first difference is about the setup costs. With positive setup times, the out of pocket cost for a cold setup is only $K_{t}$ and there is no additional out of pocket cost associated with the setup time. However, there will also be a shadow price. A positive shadow price implies that a positive cold setup time in a period reduces the effective capacity resulting in a more costly schedule. Interestingly, in our case, positive setup times may decrease the overall costs by pushing the production of some demands to later periods with smaller unit variable production costs and, more importantly, by keeping the process warm at a lower cost. The second difference is about the inventory levels at the beginning and ending periods of production series. Unlike the zero setup times case, in the presence of positive cold setup times, it is possible that there is positive beginning inventory when a cold setup is done in period $t$ in a production series of an optimal production plan for problems $\widetilde{P}$ or $P$. A positive beginning inventory in a particular series also implies that there be positive ending inventories in the immediately preceding series. Hence, we can no longer argue, in general, that $I_{u-1}=I_{v-1}=0$ in a series $\Psi_{u v \mid I_{u-1}, I_{v-1}}$ as before. However, the beginning and ending inventories for a series in an optimal plan cannot have arbitrary values and some structure can be ascertained, as we discuss in the sequel.

\section{Lemma 4}

(i) If in period $t, \gamma_{t}=1$ and $S_{t}+\sum_{\ell=1}^{\mathfrak{f}_{t}} D_{t}^{\ell} \leqslant R_{t}$, then $y_{t-1}=0$ in an optimal production plan for $\widetilde{P}$ and $P$.

(ii) If in period $t, \gamma_{t}=1$ and $S_{t}+\sum_{\ell=1}^{\mathfrak{f}_{t}} D_{t}^{\ell} \leqslant R_{t}$, then $I_{u-1}=I_{v-1}=0$ in any series $\Psi_{u v \mid I_{u-1}, I_{v-1}}\left(\right.$ and $\widehat{\Psi}_{u v \mid I_{u-1}, I_{v-1}}$ ) for $1 \leqslant u<v \leqslant N$ in an optimal production plan for problem $\widetilde{P}$ (and $P$ ).

(iii) If $S_{u}+\sum_{\ell=1}^{\mathfrak{f}_{u}} D_{u}^{\ell} \leqslant R_{u}$, then series $\Psi_{u v}\left(\right.$ and $\left.\widehat{\Psi}_{u v}\right)$ in an optimal production plan for problem $\widetilde{P}$ (and $P$ ) with positive cold setup times is identical to $\Psi_{u v}^{\prime}$ for problem $\widetilde{P}^{\prime}$ (and $\left.P^{\prime}\right)$ where $S_{u}^{\prime}=0, Q_{u}^{\prime}=Q_{u}$ and $R_{u}^{\prime}=R_{u}$ ceteris paribus.

Proof is by contradiction (Berk et al., 2006). The above lemma implies that the solution to problem $\widetilde{P}$ (and $P$ ) with positive cold setup times can be obtained by considering modified problems $\widetilde{P}^{\prime}\left(\right.$ and $P^{\prime}$ ) and the results developed in the previous subsection when there is enough capacity, in a period with a cold setup, to satisfy production of all of its demand in that period and the setup time.

Next, we consider plans where a cold setup period may not have enough capacity to do a setup and production to satisfy all of its demands. When cold setup times are zero, the Wagner-Whitin-type costs ensure that cold setups are less costly later in the horizon than early on, and it is best to produce for a particular period's demand in a period as close to it as possible. This motivates the postponement of production in the problem. However, if we only retain the Wagner-Whitin-type cost structure for positive setup times, we can no longer guarantee this postponement. We need some additional conditions on setup times and other relevant costs in the problem; but, the shadow price of a cold setup is clearly a function of the series generated within the problem and cannot be a priori defined. First, to ensure that the effective cold setup costs are nonincreasing in $t$, we make the additional reasonable assumption that $S_{t}$ is non-increasing in $t$. That is, postponing a cold setup results in a lower out-of-pocket cost and loss of capacity. Furthermore, we suggest the following sufficient condition on costs:

Condition 1. $K_{t+1}+S_{t+1}\left(c_{t}+h_{t}-c_{t+1}\right)<K_{t}-\omega_{t-1}\left(R_{t-1}-Q_{t-1}\right)$ for $1<t<N$.

This condition coupled with the previously assumed cost structure, now, ensures that there is no speculative motive, i.e. encourages postponement of production to a period as late as possible. With the extended Wagner-Whitin-type cost structure, we can now provide some further structural results on the problem with positive setup times.

As in the case of zero setup times, in an optimal plan for problem $\widetilde{P}$ with positive setup times, the production quantity in a period immediately preceding a cold setup period must be less than its warm threshold. We state this result in the following lemma. 
Lemma 5. Suppose Condition 1. A production plan $(X, A, \Gamma)$ with $S_{t} \gamma_{t}+x_{t} \geqslant Q_{t}$ and $\gamma_{t+1}=1$ for any $t$ $(1 \leqslant t<N)$ cannot be optimal for problem $P$.

Theorem 5. Suppose Condition 1. For problem $P$, in an optimal production plan $(X, A, \Gamma)$, if $\gamma_{t+1}=1$, then $y_{t}=\left[\sum_{\ell=1}^{\mathfrak{f}_{t+1}}\left(D_{t+1}^{\ell}-a_{t+1}^{\ell}\right)-\left(R_{t+1}-S_{t+1}\right)\right]^{4}$ for any $t(1 \leqslant t<N)$.

The proof is in Appendix. Note that the last two results are also valid for a full commitment problem $\widetilde{P}$, as can be ascertained from their proofs with the additional constraint that $\mathbf{A}=\mathbf{0}$.

The production schedule for a full commitment series $\Psi_{u v \mid I_{u-1}, I_{v-1}}$ feasible for problem $\widetilde{P}$ with positive beginning and ending inventories in the presence of positive setup times is identical to that of another feasible full commitment series with zero setup times and zero beginning and ending inventories which is obtained by adjusting demands, capacities and warm process thresholds. That is, there exists an equivalence property which allows us to transform a feasible series with positive beginning and ending inventory and positive setup times into another feasible full commitment series with zero beginning and ending inventory and zero setup times. This key property enables us to use only those series feasibly generated with zero setup times for the solution of problem $\widetilde{P}$ with positive setup times. To show this, we shall employ a demand modification scheme for periods in which there is a cold setup and their immediate neighbors. We introduce the following notation. Define $\mathbf{T}_{j-1}=\left\{T_{j-1}^{1} \cup T_{j-1}^{2} \cup \cdots \cup T_{j-1}^{\bar{k}_{j}}\right\}, T_{j-1}^{i}=D_{j}^{i}$ for $1 \leqslant i<\bar{k}_{j}, T_{j-1}^{\bar{k}_{j}}=G_{j}^{\bar{k}_{j}}$ with $G_{j}^{\bar{k}_{j}} \subset D_{j}^{\bar{k}_{j}}$ and $\left|G_{j}^{\bar{k}_{j}}\right|=\left[\sum_{\ell=1}^{\mathfrak{f}_{j}} D_{j}^{\ell}-R_{j}+S_{j}-\sum_{\ell=1}^{\bar{k}_{j}-1} D_{j}^{\ell}\right]^{+} \quad$ and $\quad \bar{k}_{j}=\min \left(k_{j}: \sum_{\ell=1}^{k_{j}} D_{j}^{\ell} \geqslant \sum_{\ell=1}^{\mathfrak{f}_{j}} D_{j}^{\ell}-R_{j}+S_{j}\right) \quad$ if $\quad \sum_{\ell=1}^{\mathfrak{f}_{j}} D_{j}^{\ell}-R_{j}$ $+S_{j}>0 \forall j$. Otherwise, $\bar{k}_{j}=0$ and $\mathbf{T}_{j-1}=\emptyset$. Note that, in the above construction, the demands of period $j$ are appended onto the demands of period $j-1$ starting with the classes with the highest unit revenues. The highest revenue classes are appended first to ensure the highest profit margin on the demands produced in period $j$. Now, we formally state the equivalence result. (Proof is in Appendix.)

Proposition 2 (Series equivalence). Suppose Condition 1. Let $\Psi_{u v \mid I_{u-1}, I_{v-1}}$ be a full commitment series proposed for problem $\widetilde{P}$ where $I_{u-1}=\left[\sum_{\ell=1}^{\mathfrak{f}_{u}} D_{u}^{\ell}-R_{u}-S_{u}\right]^{+}, I_{v-1}=\left[\sum_{\ell=1}^{\mathfrak{f}_{v}} D_{v}^{\ell}-R_{v}-S_{v}\right]^{+}$and $S_{u}, S_{v}>0$ with given demands $\mathbf{D}_{t}$ over periods $u$ through $v-1$. Let $\Psi_{u v \mid 0,0}^{\prime}$ be another full commitment series over periods $u$ through $v-1$ and a fictitious period $v^{\prime}$ inserted between $v-1$ and $v$ where $\mathbf{D}_{v^{\prime}}^{\prime}=\mathbf{T}_{v-1}, \mathbf{D}_{u}^{\prime}=\mathbf{D}_{u} \backslash \mathbf{T}_{u-1}, \mathfrak{f}_{v^{\prime}}^{\prime}=\bar{k}_{v}$, $\mathfrak{£}_{u}^{\prime}=£_{u}-\bar{k}_{u}+\mathbf{1}\left[\sum_{\ell=1}^{\bar{k}_{u}} D_{u}^{\ell}<\sum_{\ell=1}^{\mathfrak{f}_{u}} D_{u}^{\ell}-R_{u}+S_{u}\right], \quad p_{v^{\prime}}^{i \prime}=p_{v}^{i} \quad$ for $\quad 1 \leqslant i<\bar{k}_{v^{\prime}}, \quad \mathbf{D}_{t}^{\prime}=\mathbf{D}_{t} \quad$ for $\quad u<t \leqslant v-1$, $R_{u}^{\prime}=R_{u}-S_{u}, Q_{u}^{\prime}=Q_{u}-S_{u}, S_{u}^{\prime}=0$ with $R_{v^{\prime}}$ and $Q_{v^{\prime}}$ set arbitrarily equal to $R_{v}$ and $Q_{v}$. If $x_{v-1}<Q_{v-1}$ in the optimal schedule for $\Psi_{u v \mid 0,0}^{\prime}$, then $\Psi_{u v \mid I_{u-1}, I_{v-1}}$ is feasible, and the optimal production in periods $u$ through $v-1$ of $\Psi_{u v \mid I_{u-1}, I_{v-1}}$ and of $\Psi_{u v \mid 0,0}^{\prime}$ are identical and the two series have identical costs. Otherwise, $\Psi_{u v \mid I_{u-1}, I_{v-1}}$ cannot be optimal for problem $P$.

Note that, due to the adjustments defined in the above equivalence proposition for a period with cold setup, we can retain, in the presence of positive setup times, the definition of the economic bound for zero setup times given in Lemma 2. Based on the above findings, we have

\section{Lemma 6}

(i) Suppose Condition 1. For problem $P$ with one demand class for period $t+1$, in an optimal production plan $(X, A)$, if $\gamma_{t+1}=1$, either $y_{t}=0$ or $y_{t}=\left[D_{t+1}-\left(R_{t+1}-S_{t+1}\right)\right]^{+}$for any $t(1 \leqslant t<N)$.

(ii) Suppose Condition 1. For problem $P$ with more than one demand class for period $t+1$, in an optimal production plan $(X, A)$, if $\gamma_{t+1}=1,0 \leqslant y_{t} \leqslant\left[D_{t+1}-\left(R_{t+1}-S_{t+1}\right)\right]^{+}$for any $t(1 \leqslant t<N)$.

(iii) Suppose Condition 1. For problem $P$, in an optimal plan $\left(1-\gamma_{t}\right) \cdot x_{t} \cdot a_{t}^{\ell}=0 \forall \ell, t$.

Proof is in Appendix. At this point, it may be useful to summarize our approach and findings. In this section, we examine the properties of the optimal solution to the dynamic lot-sizing problem with immediate lost sales in the presence of positive setup times. So far, we have obtained the conditions on the feasibility of a full commitment series with positive setup times and beginning and ending inventories which may be positive. We have also established in the last key proposition that any such feasible series can be converted into an equivalent full commitment series with zero beginning and ending inventories and zero setup times. In the previous 
section, we had already established that the solution to problem $P$ in the absence of setup times consists of lost-sales-improved series with zero inventories which are obtained from full commitment series with zero inventories via lost sales decisions. Thus, we are ready to state the following (as proved in Appendix).

Lemma 7. Suppose Condition 1. The optimal solution for $P$ where $S_{t} \geqslant 0 \forall t$ consists only of series that are the lost-sales-improvements of the series for $\widetilde{P}$ with $S_{t}=0 \forall t$.

We illustrate the proposed forward solution methodology for positive cold setup times in the following example.

Example. We revisit the example in the previous section with $S_{t}=35 \forall t$. Note that the effective production capacity in a cold setup period is now 65 . This changes the production series generated, as well (see below $N=3$ or 4 ). We first illustrate the full commitment problem, $\widetilde{P}$.

$$
\begin{aligned}
& N=1 ; \quad \Psi_{1,1}=\{42\} ; \mathbf{X}_{1}=\left\{\Psi_{1,1}\right\} ; X_{1}^{*}=\Psi_{1,1} ; f_{1}^{1}=110, \\
& N=2 ; \quad \Psi_{1,2}=\{62,0\}, \Psi_{2,2}=\{20\} ; \mathbf{X}_{2}=\left\{\Psi_{1,2},\left(X_{1}^{*} \cup \Psi_{2,2}\right)\right\} ; X_{2}^{*}=\Psi_{1,2} ; f_{1}^{2}=130 \\
& N=3 ; \quad \Psi_{1,3}=\{65,37,0\}, \Psi_{2,3}=\{60,0\}, \Psi_{3,3}=\{40\} ; \mathbf{X}_{3}=\left\{\Psi_{1,3},\left(X_{1}^{*} \cup \Psi_{2,3}\right),\left(X_{2}^{*} \cup \Psi_{3,3}\right)\right\} ; \\
& \quad X_{3}^{*}=\Psi_{1,3} ; f_{1}^{3}=173, \\
& N=4 ; \quad \Psi_{1,4}=\{65,77,0,0\}, \Psi_{2,4}=\{65,35,0\}, \Psi_{3,4}=\{65,15\}, \Psi_{4,4}=\{40\} ; \\
& \mathbf{X}_{4}=\left\{\Psi_{1,4},\left(X_{1}^{*} \cup \Psi_{2,4}\right),\left(X_{2}^{*} \cup \Psi_{3,4}\right),\left(X_{3}^{*} \cup \Psi_{4,4}\right)\right\} ; X_{4}^{*}=\left(X_{2}^{*} \cup \Psi_{3,4}\right)=\{65,77,0,0\} ; f_{1}^{4}=253, \\
& N=5 ; \quad \Psi_{1,5}=\{65,70,72,0,0\}, \Psi_{2,5}=\{65,70,30,0\}, \Psi_{3,5}=\{65,80,0\}, \Psi_{4,5}=\{65,40\}, \Psi_{5,5}=\{65\} ; \\
& \mathbf{X}_{5}=\left\{\Psi_{1,5},\left(X_{1}^{*} \cup \Psi_{2,5}\right),\left(X_{2}^{*} \cup \Psi_{3,5}\right),\left(X_{3}^{*} \cup \Psi_{4,5}\right),\left(X_{4}^{*} \cup \Psi_{5,5}\right)\right\} ; X_{5}^{*}=\left(X_{2}^{*} \cup \Psi_{3,5}\right)=\{65,37,0,65,40\} ; f_{1}^{5}=308
\end{aligned}
$$

Next, we illustrate the forward solution methodology for problem $P$. In its solution we shall use the full commitment series developed above and improve them through lost sales decisions if possible. In cases of indifference, we use the lost-sales-improved series. We do not report $\mathbf{A}_{N}$ for brevity which can be obtained easily from $\mathbf{X}_{N}$

$$
\begin{aligned}
& N=1 ; \quad \Psi_{1,1}=\{42\} ; \widehat{\Psi}_{1,1}=\{0\} ; \mathbf{X}_{1}=\left\{\widehat{\Psi}_{1,1}\right\} ; X_{1}^{*}=\widehat{\Psi}_{1,1} ; f_{1}^{1}=84, \\
& N=2 ; \quad \Psi_{1,2}=\{62,0\}, \Psi_{2,2}=\{20\} ; \widehat{\Psi}_{1,2}=\{62,0\}, \widehat{\Psi}_{2,2}=\{0\} ; \\
& \mathbf{X}_{2}=\left\{\widehat{\Psi}_{1,2},\left(X_{1}^{*} \cup \widehat{\Psi}_{2,2}\right\} ; X_{2}^{*}=\left(X_{1}^{*} \cup \widehat{\Psi}_{2,2}\right)=\{0,0\} ; f_{1}^{2}=124,\right. \\
& N=3 ; \quad \Psi_{1,3}=\{65,37,0\}, \Psi_{2,3}=\{60,0\}, \Psi_{3,3}=\{40\} ; \widehat{\Psi}_{1,3}=\{65,37,0\}, \widehat{\Psi}_{2,3}=\{0,0\}, \widehat{\Psi}_{3,3}=\{0\} ; \\
& \mathbf{X}_{3}=\left\{\widehat{\Psi}_{1,3},\left(X_{1}^{*} \cup \widehat{\Psi}_{2,3}\right),\left(X_{2}^{*} \cup \widehat{\Psi}_{3,3}\right)\right\} ; X_{3}^{*}=\widehat{\Psi}_{1,3} ; f_{1}^{3}=173, \\
& N=4 ; \quad \Psi_{1,4}=\{65,77,0,0\}, \Psi_{2,4}=\{65,35,0\}, \Psi_{3,4}=\{65,15\}, \Psi_{4,4}=\{40\} ; \widehat{\Psi}_{1,4}=\{65,37,0,0\}, \\
& \widehat{\Psi}_{2,4}=\{0,0,0\}, \widehat{\Psi}_{3,4}=\{65,15\}, \widehat{\Psi}_{4,4}=\{0\} ; \\
& \mathbf{X}_{4}=\left\{\widehat{\Psi}_{1,4},\left(X_{1}^{*} \cup \widehat{\Psi}_{2,4}\right),\left(X_{2}^{*} \cup \widehat{\Psi}_{3,4}\right),\left(X_{3}^{*} \cup \widehat{\Psi}_{4,4}\right)\right\} ; X_{4}^{*}=\left(X_{2}^{*} \cup \widehat{\Psi}_{3,4}\right)=\{65,37,0,0\} ; f_{1}^{4}=253, \\
& N=5 ; \quad \Psi_{1,5}=\{65,70,72,0,0\}, \Psi_{2,5}=\{65,70,30,0\}, \Psi_{3,5}=\{65,80,0\}, \Psi_{4,5}=\{65,40\}, \Psi_{5,5}=\{65\} ; \\
& \widehat{\Psi}_{1,5}=\{65,70,72,0,0\}, \widehat{\Psi}_{2,5}=\{65,70,30,0\}, \widehat{\Psi}_{3,5}=\{65,80,0\}, \widehat{\Psi}_{4,5}=\{65,40\}, \widehat{\Psi}_{5,5}=\{65\} ; \\
& \mathbf{X}_{5}=\left\{\widehat{\Psi}_{1,5},\left(X_{1}^{*} \cup \widehat{\Psi}_{2,5}\right),\left(X_{2}^{*} \cup \widehat{\Psi}_{3,5}\right),\left(X_{3}^{*} \cup \widehat{\Psi}_{4,5}\right),\left(X_{4}^{*} \cup \widehat{\Psi}_{5,5}\right)\right\} ; X_{5}^{*}=\left(X_{2}^{*} \cup \widehat{\Psi}_{3,5}\right)=\{65,37,0,65,40\} ; f_{1}^{5}=308 .
\end{aligned}
$$

\subsection{A special case}

In the previous subsections, we have considered the case $c_{t}+h_{t}-\omega_{t}>c_{t+1}$. In this subsection, we relax this assumption for a special case. Specifically, we assume that $c_{t}=c, h_{t}=h, \omega_{t}=\omega \forall t$, and $\omega>h$. This structure still ensures that Wagner-Whitin cost structure $\left(c_{t}+h_{t}>c_{t+1}\right)$ holds. However, $\omega>h$ implies that the most economical way to keep the process warm is by producing up to the capacity in a period. That is, the warm process threshold effectively becomes $R_{t}$. Consequently, the economic bound in Lemma 2 becomes the period's capacity itself, and effectively disappears. This results in a slight change in the structure of a production series in an optimal plan for the full commitment problem as stated below.

Corollary 2. Suppose $c_{t}=c, h_{t}=h, \omega_{t}=\omega \forall t$, and $\omega>h$. In an optimal solution to problem $\widetilde{P}$, each production series $\Psi_{u v}$ is of the form 
(a) $x_{t}=R_{t}$ for $u \leqslant t \leqslant m-1$ and $m-u>0$,

(b) $x_{m}=\left[\sum_{i=m}^{v-1} \sum_{\ell=1}^{f i} D_{i}^{\ell}-y_{m-1}\right]^{+}$and $m-u \geqslant 0$.

In this setting, since the unit production and holding costs still favor postponement of production for a period's demand to a period as close to it as possible, all of the cost argumentations followed in the previous subsections still hold. Therefore, all of the structural results above are also valid for this special case.

\section{Computational results}

The optimal solution to the problem $P$ can, theoretically, be obtained by a backward solution algorithm. However, in a backward solution algorithm, even for discrete demand or largely discretized continuous demand scenarios, the size of the state space for reasonable problem settings becomes prohibitively high. Therefore, it is essential to develop forward solution algorithms when available. The structural results in the previous section enable such an algorithm. The algorithm exploits the one given in Toy and Berk (2006) and modifies it with the addition of the lost-sales-improvement module. The complexity of the full commitment problem is known to be $\mathrm{O}\left(N^{3}\right)$ and the proposed improvement algorithm has a complexity of $\mathrm{O}\left(N^{5}\right)$.

\subsection{Illustrative numerical study}

For our numerical study, we used a test problem (RD10-all) in Aksen et al. (2003): $N=16 ; £_{t}=1 \forall t$; $\mathbf{D}=(9,10,4,11,17,13,3,16,1,29,25,16,18,34,12,5) ; R_{t}=R=45, K_{t}=K=120, c_{t}=0 \forall t ;$ for $t=1, \ldots, 8$, $h_{t}=5$ and for $t=9, \ldots, 16, h_{t}=3$; for $t=1, \ldots, 8, \breve{p}_{t}=10$ and for $t=9, \ldots, 16, \breve{p}_{t}=8$. Since our setting involves also warming and setup times, we introduced additional parameters. To study the impact of unit revenues, we consider $p_{t}=r \cdot \breve{p}_{t}$ where $r=0.5,0.75,1$ and 1.25 for all $t$. Warming cost in each period takes on values proportional to the holding cost such that $\omega_{t}=\eta \cdot h_{t}$ where $\eta \in\{0.95,0.90,0.85,0.80,0.50\}$. Warm process threshold and cold setup time take on values (rounded to the nearest integer) proportional to the capacity such that $Q_{t}=Q=\left[\xi \cdot R_{t}\right]$ and $S_{t}=S=\left[\alpha \cdot R_{t}\right]$ where $\xi \in\{0.90,0.80,0.70,0.60,0.50\}$ and $\alpha \in\{0,0.05,0.10$, $0.20,0.30,0.50\} \forall t$.

In Tables 1 and 2, we tabulate the optimal production plans for problems $\widetilde{P}$ and $P$ for different values of warm process threshold and warming cost. The case where $Q=\infty$ corresponds to the case when we allow no warming; that is, the process is always cold. We see that for this case we have the highest number of lost sales and the minimum number of production periods. The value of cold setup times has an impact on the solutions. We first discuss the case of zero setup times. The results are not sensitive to warming cost but the warm threshold has an impact on the production schedules especially in the latter half of the horizon where unit revenues

Table 1

Optimal production plans and costs $(r=1, S=0)$

\begin{tabular}{|c|c|c|c|c|c|}
\hline$Q$ & $\omega_{t} / h_{t}$ & & $X^{*}$ & $A^{*}$ & Cost \\
\hline$\infty$ & - & $P$ & $\{19,0,0,0,30,0,0,17,0,29,41,0,19,45,0,0\}$ & $\{0,0,4,11,0,0,3,0,0,0,0,0,0,0,0,5\}$ & 1267 \\
\hline \multirow[t]{4}{*}{40} & 0.8 & $\widetilde{P}$ & $\{23,0,0,11,33,0,0,17,0,40,40,8,0,40,11,0\}$ & $\{0\}$ & 1148.5 \\
\hline & & $P$ & $\{19,0,0,0,30,0,0,17,0,40,40,8,0,40,11,0\}$ & $\{0,0,4,11,0,0,3,0,0,0,0,0,0,0,0,0\}$ & 1138.5 \\
\hline & 0.5 & $\widetilde{P}$ & $\{23,0,0,11,33,0,0,17,0,40,40,8,0,40,11,0\}$ & $\{0\}$ & 1130.5 \\
\hline & & $P$ & $\{19,0,0,0,30,0,0,17,0,40,40,8,0,40,11,0\}$ & $\{0,0,4,11,0,0,3,0,0,0,0,0,0,0,0,0\}$ & 1120.5 \\
\hline \multirow[t]{4}{*}{31} & 0.8 & $\widetilde{P}$ & $\{23,0,0,11,33,0,0,17,0,31,31,26,0,34,17,0\}$ & $\{0\}$ & 1114.3 \\
\hline & & $P$ & $\{19,0,0,0,30,0,0,17,0,31,31,26,0,34,17,0\}$ & $\{0,0,4,11,0,0,3,0,0,0,0,0,0,0,0,0\}$ & 1104.3 \\
\hline & 0.5 & $\widetilde{P}$ & $\{23,0,0,11,33,0,0,17,0,31,31,26,0,34,17,0\}$ & $\{0\}$ & 1067.5 \\
\hline & & $P$ & $\{19,0,0,0,30,0,0,17,0,31,31,26,0,34,17,0\}$ & $\{0,0,4,11,0,0,3,0,0,0,0,0,0,0,0,0\}$ & 1057.5 \\
\hline \multirow[t]{4}{*}{22} & 0.8 & $\widetilde{P}$ & $\{23,0,0,11,33,0,0,17,0,29,25,34,0,34,17,0\}$ & $\{0\}$ & 1105.9 \\
\hline & & $P$ & $\{19,0,0,0,30,0,0,17,0,29,25,34,0,34,17,0\}$ & $\{0,0,4,11,0,0,3,0,0,0,0,0,0,0,0,0\}$ & 1095.9 \\
\hline & 0.5 & $\widetilde{P}$ & $\{23,0,0,22,22,0,0,17,0,29,25,22,22,24,17,0\}$ & $\{0\}$ & 1000 \\
\hline & & $P$ & $\{19,0,0,0,30,0,0,17,0,29,25,22,22,24,17,0\}$ & $\{0,0,4,11,0,0,3,0,0,0,0,0,0,0,0,0\}$ & 997.5 \\
\hline
\end{tabular}


Table 2

Optimal production plans and costs $(r=1, S=13)$

\begin{tabular}{lllll}
\hline$Q$ & $\omega / h$ & $X^{*}$ & $A^{*}$ & Cost \\
\hline$\infty$ & - & $\{19,0,0,0,30,0,0,17,0,32,32,0,32,32,0,0\}$ & $\{0,0,4,11,0,0,3,0,0,0,0,6,0,0,0,5\}$ & 1345 \\
40 & 0.8 & $\{19,0,0,0,27,6,0,17,0,29,40,21,0,32,17,0\}$ & $\{0,0,4,11,0,0,0,0,0,0,0,0,0,0,0,0\}$ & 1040.1 \\
& 0.5 & $\{19,0,0,0,27,6,0,17,0,29,40,21,0,32,17,0\}$ & $\{0,0,4,11,0,0,0,0,0,0,0,0,0,0,0,0\}$ & 1020.5 \\
31 & 0.8 & $\{19,0,0,0,18,15,0,17,0,29,41,0,18,34,17,0\}$ & $\{0,0,4,11,0,0,3,0,0,0,0,0,0,0,0,0\}$ & 1026.6 \\
& 0.5 & $\{19,0,0,0,18,15,0,17,0,29,31,10,18,34,17,0\}$ & $\{0,0,4,11,0,0,0,0,0,0,0,0,0,0,0,0\}$ & 956 \\
22 & 0.8 & $\{19,0,0,11,30,0,0,17,0,29,41,0,18,34,17,0\}$ & $\{0,0,4,0,0,0,3,0,0,0,0,0,0,0,0,0\}$ & 1023.1 \\
& 0.5 & $\{9,14,0,11,30,0,0,17,0,29,25,22,22,24,17,0\}$ & $\{0,0,0,0,0,0,3,0,0,0,0,0,0,0,0,0\}$ & 908 \\
\hline
\end{tabular}

are lower. As the warm threshold gets smaller, the overall costs also decrease, as expected. We see that the cost differential between the case of no warming allowed and the warm/cold process is quite high. This suggests that managerial policies such as employing undertimes or production technology choices allowing for variable production rates (especially ability to slow down the speed of the process) may have a significant impact on the operational costs, even in the presence of lost sales. Furthermore, the observed decrease in the number of lost sales decisions with process flexibility in terms of the warm/cold option hints at possible customer relations improvements. In the presence of positive setup times, we find the production schedules and lost sales decisions to be more sensitive to the system parameters. We especially see that the lost sales decisions and the production schedule in the last half of the horizon are most sensitive.

In Table 3, we present the impact of the unit revenues; the setup time is zero in the tabulated results. We see that the optimal production plan is very sensitive to the revenues. The impact of warm process threshold is more on the production schedules than the lost sales decisions.

Table 3

Optimal production plans with respect to changes in unit revenues $(\eta=0.95, S=0)$

\begin{tabular}{llll}
\hline$Q$ & $r$ & $X$ & $A$ \\
\hline 40 & 0.5 & $\{0,0,0,0,0,0,0,0,0,40,30,0,0,40,11,0\}$ & $\{9,10,4,11,17,13,3,16,1,0,0,0,18,0,0,0\}$ \\
& 0.75 & $\{0,0,0,0,30,0,0,17,0,40,40,8,0,40,11,0\}$ & $\{9,10,4,11,0,0,3,0,0,0,0,0,0,0,0,0\}$ \\
& 1.25 & $\{23,0,0,11,33,0,0,17,0,40,40,8,0,40,11,0\}$ & $\{0\}$ \\
31 & 0.5 & $\{0,0,0,0,0,0,0,0,0,31,31,26,0,34,17,0\}$ & $\{9,10,4,11,17,13,3,16,1,0,0,0,0,0,0,0\}$ \\
& 0.75 & $\{0,0,0,0,30,0,0,17,0,31,31,26,0,34,17,0\}$ & $\{9,10,4,11,0,0,3,0,0,0,0,0,0,0,0,0\}$ \\
22 & 1.25 & $\{23,0,0,11,33,0,0,17,0,31,31,26,0,34,17,0\}$ & $\{0\}$ \\
& 0.5 & $\{0,0,0,0,0,0,0,0,0,29,25,34,0,34,17,0\}$ & $\{9,10,4,11,17,13,3,16,1,0,0,0,0,0,0,0\}$ \\
& 0.75 & $\{0,0,0,0,30,0,0,17,0,29,25,34,0,34,17,0\}$ & $\{9,10,4,11,0,0,3,0,0,0,0,0,0,0,0,0\}$ \\
\hline
\end{tabular}

Table 4

Cost breakdown $(r=1)$

\begin{tabular}{|c|c|c|c|c|c|c|c|}
\hline$S$ & $Q$ & $\omega / h$ & Setup & Holding & Warming & Lost sales & Total cost \\
\hline \multirow[t]{4}{*}{0} & 40 & 0.9 & 600 & 318 & 42.75 & 180 & 1140.75 \\
\hline & & 0.5 & 600 & 318 & 22.5 & 180 & 1120.5 \\
\hline & 22 & 0.9 & 600 & 189 & 133.95 & 180 & 1102.95 \\
\hline & & 0.5 & 480 & 183 & 154.5 & 180 & 997.5 \\
\hline \multirow[t]{4}{*}{13} & 40 & 0.9 & 600 & 246 & 46.55 & 150 & 1042.55 \\
\hline & & 0.5 & 600 & 246 & 24.5 & 150 & 1020.5 \\
\hline & 22 & 0.9 & 600 & 183 & 216.6 & 70 & 1032.55 \\
\hline & & 0.5 & 480 & 153 & 264.5 & 30 & 908 \\
\hline
\end{tabular}


In Table 4, we report the cost breakdowns for the above results. The figures therein provide further instances of potential cost benefits of positive setup times - i.e., negative shadow prices. We see that as we change setup times from zero to thirteen, the total cost decreases. This is largely due to the decrease in lost sales and holding costs. This can be explained through the increase in the number of periods in which there is production while maintaining the same number of cold setups since the process can be kept warm more easily with the insertion of setup times. More frequent production results in lower inventories and also positive marginal profits for a larger number of periods' demands with shorter production series.

\section{Conclusions and future work}

In this work, we have considered joint lot sizing and lost sales decisions for a capacitated process which can be kept warm if the production quantity exceeds a positive threshold amount. The problem is first formulated as a DP problem. We have established the structure of the optimal production schedule and lost sales decisions for the case of zero setup times and provided a polynomial solution algorithm based on the lost-sales-improvements of the full commitment problem. We have also obtained conditions under which the case of positive setup times can be solved for optimality employing a similar improvement scheme. Although our focus has been to obtain the optimal solution and investigate its characteristics, the related issue of heuristic solutions remains an open research area.

\section{Acknowledgments}

The authors gratefully acknowledge the Editors and the three anonymous reviewers for their helpful feedback, and Dr. Aksen for providing his numerical data set.

\section{Appendix}

Proof of Proposition 1. We will prove this result in an algorithmic fashion. Consider problem $P$ in which there is at least one period where the demand exceeds the capacity. Let $t$ denote the latest of such a period. The construction of the proof rests on showing that moving the excess demand quantity in $t$ to the immediate period $t-1$ in problem $P$ results in an equivalent problem $P^{\prime}$. For period $t$, label the demand classes such that $p_{t}^{\ell-1} \geqslant p_{t}^{\ell}$ for $\ell=2, \ldots, £_{t}$. Define $\hat{\ell}=\max \left\{\ell: \sum_{j=1}^{\ell} D_{t}^{j}<R_{t}\right\}, £_{t}^{\prime}=\hat{\ell}+1, £_{t-1}^{\prime}=£_{t-1}+£_{t}-\hat{\ell}$ and $£_{j}^{\prime}=£_{j}$ for all $j \neq t-1, t$. For every $(\mathbf{X}, \mathbf{A})$ and the corresponding inventory levels $\mathbf{Y}$ feasible for $P$, define $\left(\mathbf{X}^{\prime}, \mathbf{A}^{\prime}\right)$ and $\mathbf{Y}^{\prime}$ as follows. $\mathbf{X}^{\prime}=\mathbf{X} ;\left(a_{j}^{\ell}\right)^{\prime}=a_{j}^{\ell} \forall \ell, j \neq t-1, t ;\left(a_{t-1}^{\ell}\right)^{\prime}=a_{t-1}^{\ell}$ for $\ell=1, \ldots, £_{t-1} ;\left(a_{t}^{\ell}\right)^{\prime}=a_{t}^{\ell}$ for $\ell=1, \ldots, \hat{\ell}$; $\left(a_{t-1}^{\mathfrak{f}_{t-1}+1}\right)^{\prime}+\left(a_{t-1}^{\hat{\ell}+1}\right)^{\prime}=a_{t}^{\hat{\ell}+1} ; \quad\left(a_{t-1}^{\mathfrak{f}_{t-1}+j}\right)^{\prime}=a_{t}^{\hat{\ell}+j}$ for $j=2, \ldots, \mathfrak{£}_{t}-\hat{\ell}$. Furthermore, $y_{j}^{\prime}=y_{j}$ for $j \neq t-1$ and $y_{t-1}^{\prime}=y_{t-1}-\left(\sum_{j=1}^{\mathfrak{f}_{t}} D_{t}^{j}-R_{t}\right)+\sum_{j=1}^{\mathfrak{f}_{t} \hat{\ell}}\left(a_{t-1}^{\mathfrak{t}_{t-1}+j}\right)^{\prime}$.

Now, we re-write the original problem $P$ in terms of the newly defined variables. Considering Eq. (3), we have, $\mathbf{D}_{j}^{\prime}=\mathbf{D}_{j}$ for $j \neq t-1, t$; and, $\left(D_{t-1}^{\ell}\right)^{\prime}=D_{t-1}^{\ell} \quad$ for $\ell=1, \ldots, £_{t-1} ; \quad\left(D_{t}^{\ell}\right)^{\prime}=D_{t}^{\ell}$ for $\ell=1, \ldots, \hat{\ell}$; $\left(D_{t}^{\hat{\ell}+1}\right)^{\prime}=D_{t}^{\hat{\ell}+1}-\left(R_{t}-\sum_{j=1}^{\hat{\ell}} D_{t}^{j}\right) ;\left(D_{t-1}^{\mathfrak{f}_{t-1}+1}\right)^{\prime}+\left(D_{t-1}^{\hat{\ell}+1}\right)^{\prime}=D_{t}^{\hat{\ell}+1} ;\left(D_{t-1}^{\mathfrak{t}_{t-1}+j}\right)^{\prime}=D_{t}^{\hat{\ell}+j}$ for $j=2, \ldots, \mathfrak{£}_{t}-\hat{\ell}$. Substituting the above newly defined entities in the objective function (1), and noting that $\sum_{j=1}^{\mathfrak{f}_{t}} D_{t}^{j}-R_{t}$ $=\sum_{j=1}^{\mathfrak{f}_{t-1}-\hat{\ell}}\left(D_{t-1}^{\mathfrak{f}_{t-1}+j}\right)^{\prime}$, re-arranging terms, we get we obtain the result. (For details we refer the reader to Berk et al., 2006.)

Proof of Lemma 5. Note that for feasibility of $P, y_{t} \geqslant\left[\sum_{\ell=1}^{\mathfrak{f}_{t+1}}\left(D_{t+1}^{\ell}-a_{t+1}^{\ell}\right)-\left(R_{t+1}-S_{t+1}\right)\right]^{+}$. The proof rests on developing new feasible plans that improve the given $(X, A, \Gamma)$, thereby, rendering it suboptimal. Consider a pair of periods $t$ and $t+1$ in a plan that have the posed structure.

(i) If $x_{t+1} \geqslant Q_{t+1}$, construct a plan $\overline{(X, A, \Gamma)}$ where $\bar{x}_{j}=x_{j}$ and $\bar{a}_{j}^{\ell}=a_{j}^{\ell}$ for all $j$ and $\ell ; \bar{\gamma}_{j}=\gamma_{j}$ for all $j \neq t+1$ and $\bar{\gamma}_{t+1}=0$. This schedule clearly results in a cost reduction is at least given by $K_{t+1}-\omega_{t}\left(R_{t}-\bar{x}_{t}\right)-\omega_{t+1}\left(R_{t+1}-\bar{x}_{t+1}\right) . \quad$ With Condition 1, $\quad \omega_{t+1}\left(R_{t+1}-\bar{x}_{t+1}\right)<\omega_{t+1}\left(R_{t+1}-Q_{t+1}\right)<$ 
$K_{t+2}<K_{t+1}-\omega_{t}\left(R_{t}-\bar{x}_{t}\right)$; therefore, the plan $(X, A, \Gamma)$ can be improved with a new plan which contradicts the structure posed for $t$ and $t+1$ in the lemma and, hence, cannot be optimal.

(ii) If $x_{t+1}<Q_{t+1}$ and $\gamma_{t+2}=1$, construct plan where $\bar{x}_{j}=x_{j}$ and $\bar{a}_{j}^{\ell}=a_{j}^{\ell}$ for all $j$ and $\ell$; $\bar{\gamma}_{j}=\gamma_{j}$ for all $j \neq t+1$ and $\bar{\gamma}_{t+1}=0$. Then the cost reduction is $K_{t+1}-\omega_{t}\left(R_{t}-\bar{x}_{t}\right)$, which is positive due to the assumed cost structure; therefore, the plan $(X, A, \Gamma)$ can be improved and, hence, cannot be optimal.

(iii) If $x_{t+1}<Q_{t+1}, \gamma_{t+2}=0$ and $x_{t+2} \leqslant R_{t+2}-S_{t+2}$, we construct a plan $\overline{(X, A, \Gamma)}$ where $\bar{x}_{j}=x_{j}$ and $\bar{a}_{j}^{\ell}=a_{j}^{\ell}$ for all $j$ and $\ell ; \bar{\gamma}_{j}=\gamma_{j}$ for all $j \neq t+1, t+2$ and $\bar{\gamma}_{t+1}=0$ and $\bar{\gamma}_{t+2}=1$. The resulting cost reduction is at least $K_{t+1}-K_{t+2}-\omega_{t}\left(R_{t}-\bar{x}_{t}\right)$ ( $\geqslant 0$ from Condition 1$)$; therefore, the plan $(X, A, \Gamma)$ can be improved and, hence, cannot be optimal.

(iv) If $x_{t+1}<Q_{t+1}, \quad \gamma_{t+2}=0$ and $x_{t+2}>R_{t+2}-S_{t+2}$, we construct a plan $\overline{(X, A, \Gamma)}$ where $\bar{x}_{t+1}=x_{t+1}+\left[\sum_{\ell=1}^{\mathfrak{f}_{t+1}}\left(D_{t+2}^{\ell}-a_{t+2}^{\ell}\right)-\left(R_{t+2}-S_{t+2}\right)\right]^{+}, \bar{x}_{t+2}=R_{t+2}-S_{t+2}, \bar{\gamma}_{t+1}=0$ and $\bar{\gamma}_{t+2}=1$ with all else remaining the same as in $(X, A, \Gamma)$. Note that $\left[\sum_{\ell=1}^{\mathfrak{f}_{t+1}}\left(D_{t+2}^{\ell}-a_{t+2}^{\ell}\right)-\left(R_{t+2}-S_{t+2}\right)\right]^{+} \leqslant S_{t+2} \leqslant S_{t+1}$ due to the assumed structure among setup times; hence, the plan is feasible. Moreover, this schedule yields a cost reduction at least given by $K_{t+1}-K_{t+2}-\left(h_{t+1}+c_{t+1}-c_{t+2}\right)\left(\bar{x}_{t+1}-x_{t+1}\right)-\omega_{t}\left(R_{t}-\bar{x}_{t}\right)$ $\geqslant K_{t+1}-K_{t+2}-\left(h_{t+1}+c_{t+1}-c_{t+2}\right) S_{t+2}-\omega_{t}\left(R_{t}-Q_{t}\right)>0$ from Condition 1. If $\bar{x}_{t+1}<Q_{t+1}$, we have ended up with a new plan which results in a lower cost and contradicts the posed structure in the lemma. If $\bar{x}_{t+1} \geqslant Q_{t+1}$, we end up with exactly the same setting posed in the theorem for periods $t+1$ and $t+2$. We apply the above argumentation for the period pair $t+1$ and $t+2$. Either we show (as above) that the plan $(X, A, \Gamma)$ can be improved or end up with the same setting for periods $t+2$ and $t+3$. Continuing in this fashion, either we conclude that the plan $(X, A, \Gamma)$ is not optimal before we reach the period pair $N-1$ and $N$. Construction a new production plan in which the posed conditions do not exist, the cost can be reduced by $K_{N}+\left(h_{N-1}+c_{N-1}-c_{N}-\omega_{N-1}\right)\left(R_{N-1}-\bar{x}_{N-1}\right)$.

If the newly constructed plans do not contain any pairs of the proposed structure, we stop. If not, we carry out the same modifications until all such pairs are eliminated. Hence, the result.

Proof of Theorem 5. We will prove by contradiction. Consider a plan $\overline{(X, A, \Gamma)}$ where the posed structure is violated for $y_{t}$. Clearly, if $y_{t}<\left[\sum_{\ell=1}^{\mathfrak{f}_{t+1}}\left(D_{t+1}^{\ell}-a_{t+1}^{\ell}\right)-\left(R_{t+1}-S_{t+1}\right)\right]^{+}, \overline{(X, A, \Gamma)}$ is not feasible; hence, cannot be optimal. If $t+1$ is the latest cold setup period and $y_{t}>\left[\sum_{\ell=1}^{\mathfrak{f}_{t+1}}\left(D_{t+1}^{\ell}-a_{t+1}^{\ell}\right)-\left(R_{t+1}-S_{t+1}\right)\right]^{+}>0$, one can construct a new feasible plan in which $\bar{y}_{t}=\left[\sum_{\ell=1}^{\mathfrak{f}_{t+1}}\left(D_{t+1}^{\ell}-a_{t+1}^{\ell}\right)-\left(R_{t+1}-S_{t+1}\right)\right]^{+}, x_{i} \leqslant x_{i}$ for $1 \leqslant i \leqslant t$ and $x_{i} \geqslant x_{i}$ for $t+1 \leqslant i \leqslant N$ since $\sum_{i=t+1}^{N} \sum_{\ell=1}^{\mathfrak{f}_{i}} D_{i}^{\ell} \leqslant \sum_{i=t+1}^{N} R_{i}$. This plan yields a lower cost, therefore, $\overline{(X, A, \Gamma)}$ cannot be optimal. Let $j(>t+1)$ be the next cold setup period in $\overline{(X, A, \Gamma)}$. If $\bar{y}_{t}-\left[\sum_{\ell=1}^{\mathfrak{f}_{t+1}}\left(D_{t+1}^{\ell}-a_{t+1}^{\ell}\right)-\left(R_{t+1}-S_{t+1}\right)\right]^{+}<\sum_{i=t+1}^{j-2} R_{i}-S_{t+1}-\sum_{i=t+1}^{j-1} x_{i}+Q_{j-1}$, then one can construct a new feasible plan $\overline{\overline{(X, A, \Gamma)}}$ in which $\overline{\bar{y}}_{t}=\left[\sum_{\ell=1}^{\mathfrak{f}_{t+1}}\left(D_{t+1}^{\ell}-a_{t+1}^{\ell}\right)-\left(R_{t+1}-S_{t+1}\right)\right]^{+}, \overline{\bar{x}}_{i} \leqslant x_{i}$ for $1 \leqslant i \leqslant t$ and $\overline{\bar{x}}_{i} \geqslant x_{i}$ for $t+1 \leqslant i \leqslant j-2$ and $\overline{\bar{x}}_{j-1}<Q_{t-1}$. This plan yields a lower cost; therefore $\overline{(X, A, \Gamma)}$ cannot be optimal. If not, then, one can construct a plan in which $\overline{\bar{y}}_{t}>\left[\sum_{\ell=1}^{\mathfrak{t}_{t+1}}\left(D_{t+1}^{\ell}-a_{t+1}^{\ell}\right)-\left(R_{t+1}-S_{t+1}\right)\right]^{+}, \overline{\bar{x}}_{i} \leqslant x_{i}$ for $1 \leqslant i \leqslant t$ and $\overline{\bar{x}}_{i} \geqslant x_{i}$ for $t+1 \leqslant i \leqslant j-2$ and $\overline{\bar{x}}_{j-1} \geqslant Q_{t-1}$. Noting that this plan yields a lower cost and yet can be improved further from Lemma 5 eliminating the cold setup in period $j$, we continue with the improvements by constructing new plans until either $t$ is the latest cold setup period in the plan or there exists a cold setup in period $i(\geqslant j+1)$ and $\overline{\bar{y}}_{t}=\left[\sum_{\ell=1}^{\mathfrak{f}_{t+1}}\left(D_{t+1}^{\ell}-a_{t+1}^{\ell}\right)-\left(R_{t+1}-S_{t+1}\right)\right]^{+}$. Hence, the result.

Proof of Proposition 2. If $x_{v-1}<Q_{v-1}$, this condition implies that there is no production in the fictitious period and all demands appended from period $v$ are produced prior to $v^{\prime}$. A feasible series exists where $y_{v-1}^{\prime}=0$. Clearly, the production schedule $\Psi_{u v \mid I_{u-1}, I_{v-1}}$ must then be equal to that of $\Psi_{u v \mid 0,0}^{\prime}$. Otherwise, having a cold setup in period $v$ is not feasible from Lemma 5 and $\Psi_{u v \mid I_{u-1}, I_{v-1}}$ cannot be feasible. Hence, the result. 


\section{Proof of Lemma 6}

(i) From Theorem 5, we have $y_{t}=\left[D_{t+1}-\left(R_{t+1}-S_{t+1}\right)\right]^{+}$for $\widetilde{P}$. From Proposition 1, we know that the demand in period $t$ is adjusted such that the additional demand coming from period $t+1$ via the adjustment is equal to $y_{t}$. From Theorem 4, we either keep this demand (class) or lose it entirely. Hence, the result.

(ii) With more than one class, we can no longer guarantee that all of the appended demands from period $t+1$ are lost if any is lost. However, using the same argument as in (i), each appended demand class is considered for possible lost sales individually; the result follows.

(iii) This is a relaxation of Theorem 2 in the presence of positive setup times and follows from (i) and (ii).

\section{References}

Aggarwal, A., Park, J.K., 1993. Improved algorithms for economic lot size problems. Operations Research 41 (3), $549-571$.

Agra, A., Constantino, M., 1999. Lot sizing with backordering and start-ups: The case of Wagner-Whitin costs. Operations Research Letters 25, 81-88.

Aksen, D., Altinkemer, K., Chand, S., 2003. The single-item lot-sizing problem with immediate lost sales. European Journal of Operational Research 147, 558-566.

Allahverdi, A., Gupta, J.N.D., Aldowaisan, T., 1999. A review of scheduling research involving setup considerations. Omega 27, $219-239$.

Allahverdi, A., Ng, C.T., Cheng, T.C.E., Kovalyov, M.Y., this issue. A survey of scheduling problems with setup times or costs. European Journal of Operational Research, doi:10.1016/j.ejor.2006.06.060.

Berk, E., Toy, A.Ö., Hazır, Ö., 2006. Single item lot sizing problem for a warm/cold process with immediate lost sales: Extended version with proofs. Working Paper \#2006-1, Faculty of Business Administration, Bilkent University.

Bitran, G.R., Yanasse, H.H., 1982. Computational complexity of the capacitated lot size problem. Management Science 28 (10), 1174 1186.

Brahimi, N., Dauzere-Peres, S., Najid, N.M., Nordli, A., 2006. Single item lot sizing problems. European Journal of Operational Research 168, 511-528.

Bruggemann, W., Jahnke, H., 2000. The discrete lot-sizing and scheduling problem: Complexity and modification for batch availability. European Journal of Operational Research 124, 511-528.

Chung, C., Lin, C.H.M., 1988. An $\mathrm{O}\left(T^{2}\right)$ algorithm for the $N I / G / N I / N D$ capacitated lot size problem. Management Science 34, $420-426$.

Eiamkanchanalai, S., Banerjee, A., 1999. Production lot sizing with variable production rate and explicit idle capacity cost. International Journal of Production Economics 59, 251-259.

Eppen, G.D., Martin, R.K., 1987. Solving multi-item capacitated lot-sizing problems using variable redefinition. Operations Research 35 (6), 832-848.

Fleischmann, B., 1990. The discrete lot-sizing and scheduling problem. European Journal of Operational Research 44, 337-348.

Florian, M., Klein, M., 1971. Deterministic production planning with concave costs and capacity constraints. Management Science 18 (1), $12-20$.

Gallego, G., 1993. Reduced production rates in the economic lot scheduling problem. International Journal of Production Research 31 , 1035-1046.

Gupta, D., Magnusson, T., 2005. The capacitated lot-sizing and scheduling problem with sequence-dependent setup costs and setup times. Computers and Operations Research 32, 727-747.

Hindi, K.S., 1995a. Efficient solution of the single-item, capacitated lot-sizing problem with start-up and reservation costs. Journal of Operational Research Society 46 (10), 1223-1236.

Hindi, K.S., 1995b. Solving the single-item, capacitated dynamic lot-sizing problem with startup and reservation costs by tabu search. Computers and Industrial Engineering 28 (4), 701-707.

Karimi, B., Fatemi Ghomi, S.M.T., Wilson, J.M., 2003. The capacitated lot sizing problem: A review of models and algorithms. Omega 31 (5), 365-378.

Karmarkar, U.S., Kekre, S., Kekre, S., 1987. The dynamic lot-sizing problem with startup and reservation costs. Operations Research 35 (3), 389-398.

Liu, X., Wang, C., Chu, F., Chu, C., 2004. A forward algorithm for capacitated lot sizing problem with lost sales. In: Proceedings of the 5th World Congress on Intelligent Control and Automation, June 15-19.

Loparic, M., Marchand, H., Wolsey, L.A., 2003. Dynamic knapsack sets and capacitated lot-sizing. Mathematical Programming Series B $95,53-59$.

Manne, A.S., 1958. Programming of economic lot sizes. Management Science 4 (2), 115-135.

Moon, I., Gallego, G., Simchi-Levi, D., 1991. Controllable production rates in a family production context. International Journal of Production Research 29, 2459-2470.

Pochet, Y., Wolsey, L.A., 1995. Algorithms and reformulations for lot sizing problems. DIMACS Series in Discrete Mathematics and Theoretical Computer Science 20, 245-293. 
Robinson, E.P., Sahin, F., 2001. Economic production lot sizing with periodic costs and overtime. Decision Sciences Journal 32 (3), $423-$ 452.

Sandbothe, R.A., Thompson, G.L., 1990. A forward algorithm for the capacitated lot size model with stockouts. Operations Research 38 (3), 474-486.

Silver, E.A., 1990. Deliberately slowing down output in a family production context. International Journal of Production Research $28,17-$ 27.

Toy, A.O., Berk, E., 2006. Dynamic lot sizing problem for a warm/cold process. IIE Transactions 38 (11), $1027-1044$.

Van Hoesel, C.P., Wagelmans, A.P., 1996. An O $\left(T^{3}\right)$ algorithm for the economic lot-sizing problem with constant capacities. Management Science 42, 142-150.

Wagner, H.M., Whitin, T.M., 1958. Dynamic version of the economic lot size model. Management Science 5 (1), 89-96.

Wolsey, L.A., 1995. Progress with single-item lot-sizing. European Journal of Operational Research 86, 395-401. 